\title{
Importance of k-space trajectory on Off resonance artifact in echo-planar velocity imaging
}

\author{
Jacob A Bender ${ }^{1,2^{*}}$, Orlando P Simonetti ${ }^{1,2}$ \\ From 15th Annual SCMR Scientific Sessions \\ Orlando, FL, USA. 2-5 February 2012
}

\section{Summary}

Top-down and center-out echo planar imaging (EPI) trajectories were thoroughly studied in theory, phantom scans, and volunteer scans to establish a clear understanding of the manifestation of off-resonance artifacts.

\section{Background}

EPI is a highly efficient data acquisition technique, but is sensitive to off-resonance. In cardiac and flow imaging, field inhomogeneity is typically $70 \mathrm{~Hz}$ in the myocardium and $100+\mathrm{Hz}$ in the blood pool at $1.5 \mathrm{~T}(1)$. Choice of $\mathrm{k}-$ space trajectories is important; the center-out trajectory is often recommended over top-down to minimize TE and thereby maximize signal and minimize flow and motion error accumulation. Previous work has noted higher artifact with the center-out trajectory (2) although a comprehensive and systematic description is lacking.

\section{Methods}

Theoretical point spread function (PSF) calculations and computer simulations were performed to compare the center-out and top-down EPI trajectories. A gradient echo planar sequence (GRE-EPI) was developed with through plane two-sided (symmetric) velocity encoding and an echo time of $2.2 \mathrm{~ms}$ (center-out) and $6.3 \mathrm{~ms}$ (topdown). Shared velocity encoding (SVE) was used to reconstruct flow images (3). A constant flow phantom was imaged matching clinical image parameters. Demonstrative scans at the aortic valve in a single volunteer were preformed. In both phantom and volunteer scans, a frequency offset applied to investigate offresonance effects.

\section{Results}

PSF analysis and computer simulations revealed that offresonance causes a simple positional shift with topdown trajectory while the center-out trajectory leads to a more severe and complex artifact comprised of a positional shift, splitting, and blurring (see Figure). The distance of the shift artifact is twice as great with the center-out trajectory compared to top-down.

The top-down trajectory does not modulate the phase of the signal whereas the center-out trajectory does. This in combination with the phase effects from velocity encoding leads to complex artifacts affecting both the magnitude and phase image.

For the center-out trajectory, artifact phase modulation and velocity encoding leads to differences in magnitude images from the positive and negative velocity encoded $\mathrm{k}$-spaces. This can cause a severe flickering the in the magnitude cines in the presence of flow and offresonance.

The center-out trajectory provided a $15.6 \%$ higher signal than the top-down trajectory attributable to the shorter TE.

Flow quantification is overestimated and peak velocity suprizingly well maintained (Table 1).

\section{Conclusions}

A center-out EPI trajectory produces a more complex, severe, and variable artifact than a top-down trajectory with only a moderate improvement in the signal level.

\section{Author details}

'Department of Biomedical Engineering, The Ohio State University, Columbus, OH, USA. ${ }^{2}$ Dorothy M. Davis Heart \& Lung Research Institute, The Ohio State University, Columbus, OH, USA.

Published: 1 February 2012

'Department of Biomedical Engineering, The Ohio State University,

Columbus, OH, USA

Full list of author information is available at the end of the article

( 2012 Bender and Simonetti; licensee BioMed Central Ltd. This is an open access article distributed under the terms of the Creative 


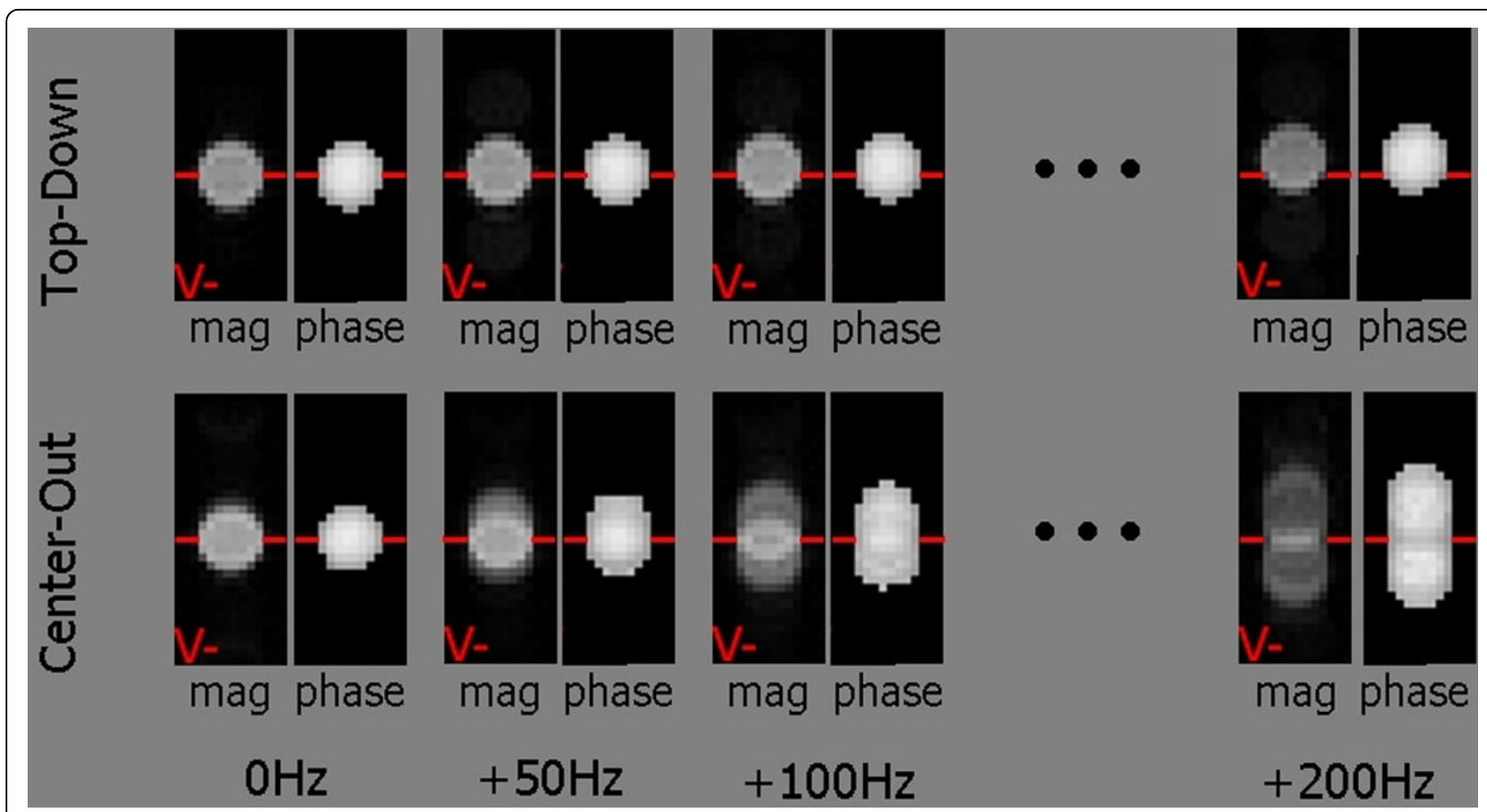

Figure 1 Magnitude and phase images for both trajectories for various off resonances. Phase images are magnitude threshold masked. Shift, splitting, and blurring artifacts were easily seen with the center-out trajectory and were not appreciable for the top-down trajectory in all experiments.

Table 1 Peak velocity and flow quantification.

\begin{tabular}{|c|c|c|c|c|c|}
\hline & Trajectory & Top-Down & Top-Down & Center-Out & Center-Out \\
\hline & Off Resonance & $\mathrm{OHz}$ & $100 \mathrm{~Hz}$ & $\mathrm{OHz}$ & $100 \mathrm{~Hz}$ \\
\hline \multirow[t]{3}{*}{ Peak Velocity $(\mathrm{cm} / \mathrm{s})$} & Simulation & 135.7 & 135.7 (no change) & 135.7 & $129.8(+11.7 \%)$ \\
\hline & Phantom & 137.5 & $138.8(+0.9 \%)$ & 138.5 & $133.2(-3.8 \%)$ \\
\hline & Volunteer & 140.4 & $146.3(+4.2 \%)$ & 134.3 & 147.6 (+9.9\%) \\
\hline Flow $(\mathrm{ml} / \mathrm{s})$ & Phantom & 484.7 & $487.1(+0.5 \%)$ & 496.3 & 838.7 (+69\%) \\
\hline
\end{tabular}

Flow quantification for the off resonant center-out trajectory due to the spreading of the velocity over a larger ROI. The peak velocity is surprisingly only slightly underestimated with off-resonant center-out trajectory. Phantom experiments with an ideally homogenous signal intensity showed the magnitude signal intensity standard deviation didn\&\#8217t increase greatly with off-resonance top-down trajectory $(5.2 @ 0 \mathrm{~Hz}$ to $8.3 @ 100 \mathrm{~Hz})$ while it did for the center-out trajectory (13.0@0Hz to 39.3@100Hz).

\section{References}

1. Reeder : MRM. 1998, 39:988-998.

2. Luk-Pat : MRM. 1997, 37:3,436-447.

3. Lin , Hung-Yu : Shared Velocity Encoding (SVE): A method to improve

the temporal resolution of phase contrast velocity measurements. MRM

doi:10.1186/1532-429X-14-S1-W68

Cite this article as: Bender and Simonetti: Importance of k-space trajectory on Off resonance artifact in echo-planar velocity imaging. Journal of Cardiovascular Magnetic Resonance 2012 14(Suppl 1):W68.

\section{Submit your next manuscript to BioMed Central} and take full advantage of:

- Convenient online submission

- Thorough peer review

- No space constraints or color figure charges

- Immediate publication on acceptance

- Inclusion in PubMed, CAS, Scopus and Google Scholar

- Research which is freely available for redistribution 